\title{
Comparison of Selected Methods for Radial Velocity Measurements
}

\author{
Štefan Parimucha ${ }^{1,2}$ and Petr Škoda ${ }^{1}$ \\ ${ }^{1}$ Astronomical Institute, Academy of Sciences of the Czech Republic, Ondřejov \\ email: skoda@sunstel.asu.cas.cz \\ ${ }^{2}$ Institute of Physics, Faculty of Natural Sciences, Šafárik University, Košice, Slovakia, \\ email: parimuch@ta3.sk
}

\begin{abstract}
We present a comparison of selected methods for measuring radial velocities in stellar spectra. We compare cross-correlation, line-profile fitting with Gauss, Lorentz and Voigt functions and a less-known mirroring method. We discuss their applicability and precision and indicate their advantages and disadvantages. The mirroring method proved to be useful for the analysis of Be stars, but is not implemented in any major astronomical packages.
\end{abstract}

Keywords. spectroscopy, radial velocity measurements

\section{Introduction}

The measurement of radial velocities (RVs) of absorption and emission spectral lines is a fundamental task of spectral analysis. Precise RVs are obligatory for the determination of the orbital elements of binaries, analysis of pulsations of stars, as well as detection of extrasolar planets. The precision of the RV determination depends on the calibration of the spectrum and method used for RV measurements.

Most of the current astronomical packages used for the analysis of stellar spectra (e.g., IRAF, MIDAS, SpecView, Starlink) use one of the following methods for estimating RVs:

- The cross-correlation method is based on the correlation between an analysed spectrum and some template with known RVs in a standard system, e.g., spectra of RV standard stars, synthetic spectra based on stellar atmosphere models, or even the spectrum of the object at a different time (measurement of differential RVs)

- Fitting of line profiles with Gauss, Lorentz or Voigt functions, when we have to assume some shape of the spectral line profile and select an interval for the fitting. This method is demonstrated in Figure 1 on a slightly asymmetric line profile of HeI $6678 \AA$ of the Be star HD 6226. The resulting RVs are shown in Figure 1 as well.

\section{Mirroring method}

One of the less known and used method for determining RVs is comparing the direct and flipped images of the line profiles, the so-called mirroring or oscilloscopic method. It was originally used in analogue oscilloscopic comparators. This interactive method was successfully implemented and used in the SPEFO package developed at the Astronomical Institute of the Academy of Sciences of the Czech Republic (for more details and program features see Škoda 1996). Later it was added to the Virtual Observatory (VO) enabled package SPLAT-VO (http://star-www.dur.ac.uk/ pdraper/splat/splat-vo/).

The principle of this method can be summarized as follows (see also Figure 2): 


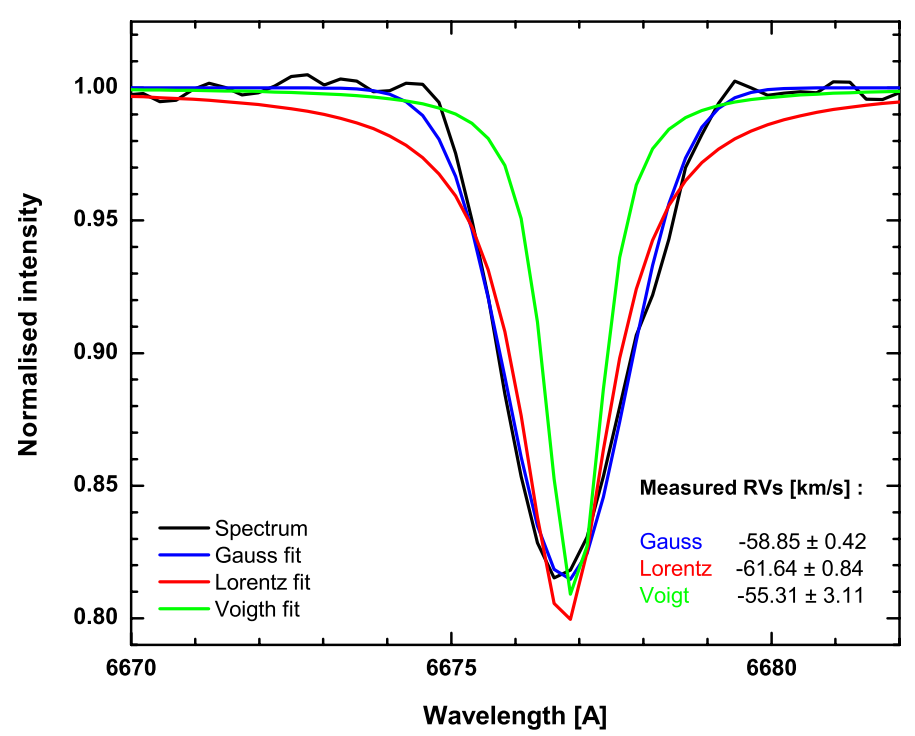

Figure 1. Fitting of the spectral line HeI $\lambda 6678$ by Gauss, Lorentz and Voigt functions with corresponding RVs

(a) create a flipped image of the line profile around the laboratory wavelength of the spectral line (Figure 2, left)

(b) move the flipped profile while both profiles (original and flipped) are overlapped as much as possible, at least in particular region, e.g., for the core of the line (Figure 2, middle)

(c) same as in (b) but for the wings of the line (Figure 2, right)

\section{Comparison of the methods}

We tested line-profile fitting and mirroring methods on the slightly asymmetric $\lambda 6678 \AA$ line of HeI. The resulting RVs are displayed in Figures 1 and 2.

We note a big difference between RVs determined by the particular fits of the line profile. The accuracy of this method strongly depends on the symmetry of the spectral line and used function.
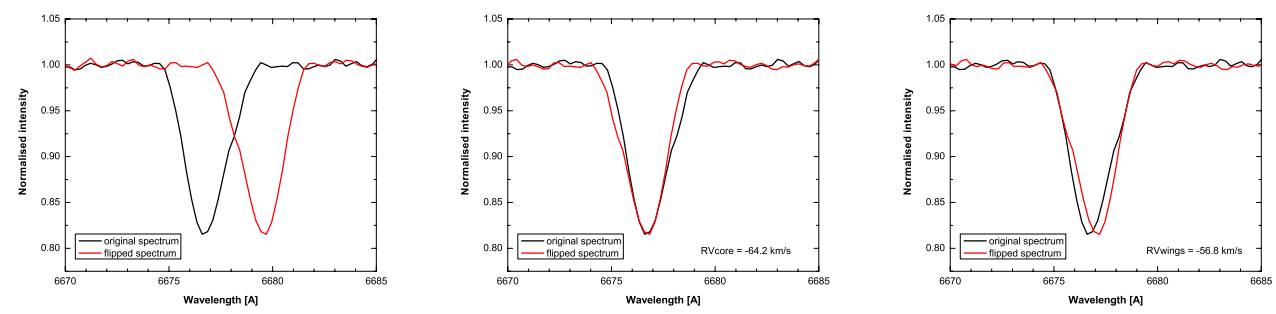

Figure 2. The principle of the mirroring method 


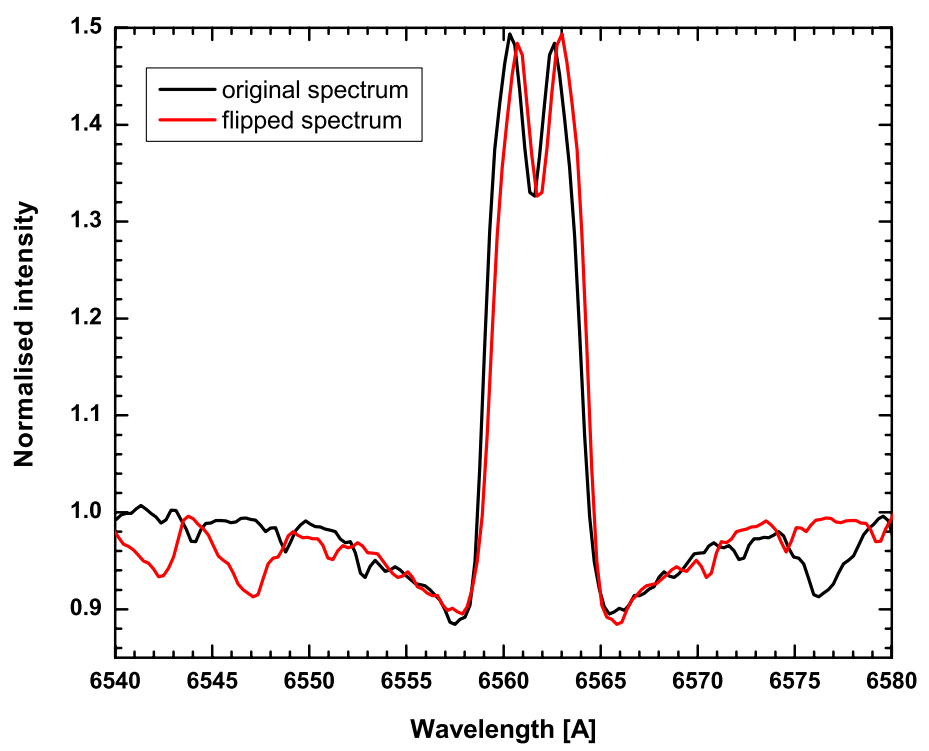

Figure 3. Application of the mirroring method on complicated line profile of H $\alpha$ of HD 6226 .

Table 1. The advantages and disadvantages of RVs measurements methods.

\begin{tabular}{l|l|l}
\hline Method & + & - \\
\hline Cross-correlation & $\begin{array}{l}\text { - very effective for a good } \\
\text { template }\end{array}$ & $\begin{array}{l}\text { - necessity to have a good } \\
\text { template }\end{array}$ \\
\hline Fitting line profile & $\begin{array}{l}\text { - works well on symmetric line } \\
\text { profiles } \\
\text { - easy implementation }\end{array}$ & $\begin{array}{l}\text { - works well only on symmetric } \\
\text { line profiles and the accuracy } \\
\text { depends on the function used } \\
\text { - cannot measure RV in different } \\
\text { parts of the profile (especially } \\
\text { in the wings) }\end{array}$ \\
\hline Mirroring line profile & $\begin{array}{l}\text { - can measure RV in different } \\
\text { parts of the line profile } \\
\text { (core, wings) } \\
\text { - very effectively can measure } \\
\text { RV of asymmetric and } \\
\text { complicated line profiles } \\
\text { (e.g., in Be stars, Figure 3) }\end{array}$ & $\begin{array}{l}\text { - relatively strong subjective } \\
\text { factors - experience dependent } \\
\text { not implemented in major } \\
\text { astronomical packages }\end{array}$ \\
\hline \multirow{2}{*}{}
\end{tabular}

The advantage of the mirroring method is the possibility of estimating RVs at several different parts of the profile, giving a quantitative measure of line profile variability. It resembles the method of bi-sectors.

This method was successfully used in the analysis of many objects with asymmetric and complex line profiles, especially Be and B[e] stars, e.g., HD 6226, (Bozic et al. 2004), $\epsilon$ Per, (Libich et al. 2006), 59 Cyg, (Harmanec et al. 2002) and many others.

The advantages and disadvantages of each method are summarized in Table 1. 


\section{Acknowledgements}

We used spectra of HD 6226 obtained by 2-m telescope at Ondřejov Observatory of the Academy of Sciences of the Czech Republic. This work was supported by Slovak Academy of Sciences VEGA grant No. 4014, grant of Šafárik University VVGS 10/2006 and grant of GČR 205/06/0584. The first author would like to thank the LOC for support.

\section{References}

Bozic, H., Harmanec, P., Yang, S., et. al. 2004, A\&SA 416, 669

Harmanec, P., Bozic, H., Percy, J.R., et al.. 2002, A\&A 387, 580

Libich, J., Harmanec, P., Vondrák, J., et al. 2006, A\&A 446, 583

Škoda, P. 1996, ASPC 101, 187 